\title{
Trait evolution in adaptive radiations: modelling and measuring interspecific competition on phylogenies
}

$1 \quad$ Article manuscript

2 Keywords: adaptive radiation, character displacement, phylogenetics, ecological modelling 


\section{Abstract}

The incorporation of ecological processes into models of trait evolution is important for understanding past drivers of evolutionary change. Species interactions have long been thought to be key drivers of trait evolution. However, models for comparative data that account for interactions between species are lacking. One of the challenges is that such models are intractable and difficult to express analytically. Here we present phylogenetic models of trait evolution that includes interspecific competition amongst species. Competition is modelled as a tendency of sympatric species to evolve towards distinct niches, producing trait overdispersion and high phylogenetic signal. The model predicts elevated trait variance across species and a slowdown in evolutionary rate both across the clade and within each branch. The model also predicts a reduction in correlation between otherwise correlated traits. We used an Approximate Bayesian Computation (ABC) approach to estimate model parameters. We tested the power of the model to detect deviations from Brownian trait evolution using simulations, finding reasonable power to detect competition in sufficiently large $(20+$ species $)$ trees. We applied the model to examine the evolution of bill morphology of Darwin's finches, and found evidence that competition affects the evolution of bill length. 


\section{Introduction}

2 There is an increasing drive to combine evolutionary and ecological perspectives in order to

3 fully capture the long-term dynamics of ecological communities (Johnson \& Stinchcombe

4 2007, Cavender-Bares et al. 2009, Schoener 2011, Pennell and Harmon 2013, Hadfield et al.

5 2014, Price et al. 2014, Pigot and Etienne 2015). This has led to insights into the roles of

6 ecological processes such as competitive exclusion and character displacement in shaping

7 distributions of traits (Webb et al. 2002, Kraft et al. 2007, Emerson and Gillespie 2008,

8 Vamosi et al. 2009). However linking such patterns in data to underlying processes is

9 difficult, since any given pattern could be the outcome of several processes (Dayan and

10 Simberloff 2005, Mayfield and Levine 2010).

11 Evidence that competition has shaped trait evolution has been generated using two

12 main approaches. This first is the observation of character displacement, i.e. a tendency for

13 species with overlapping ranges to exhibit increased phenotypic differences where they

14 coexist (Schluter and McPhail 1992, Dayan and Simberloff 2005, Pfennig and Pfennig 2010,

15 Stuart \& Losos 2013). The second source of evidence for competitive effects makes use of a

16 phylogeny to measure the distribution of species trait values relative to a null model (Webb et

17 al. 2002, Freckleton and Harvey 2006, Vamosi et al. 2009). This is especially useful for

18 adaptive radiations, where typically several similar species are confined to the same

19 geographical area. Distributions that are more even than expected by chance (Webb et al.

20 2002, Dayan and Simberloff 2005, Davies et al. 2012) are taken as evidence that past

21 competition caused species to seek unique ecological niches.

22 Convergent evolution of sets of species in separate clades has also been observed and

23 interpreted as evidence of interspecific competition (Moen and Wiens 2009). With close

24 niche packing interspecific competition can reduce evolutionary rates, even with a changing

25 environment (De Mazencourt et al. 2008). Phylogenetic comparative models of adaptive

26 radiations have slowing evolutionary rates, implicitly assuming that competition for finite 
1 niche space is an underpinning mechanism (e.g. the 'early burst' model, Harmon 2010a).

2 Despite much study, however, the importance of competition remains uncertain (Gillespie

3 2001, Cavender-Bares et al. 2009) and, importantly, direct tests for evidence of past

4 competition in phylogenetic data are lacking.

$5 \quad$ One approach could be to explicitly model the evolution of traits in systems of species

6 in which competition is occurring. In general evolutionary models use some combination of

7 continuous random change through time (Felsenstein 1973), possibly with changes of rate

8 (Garland et al. 1992, Pagel 1997, Freckleton et al. 2002, Blomberg et al. 2003, Eastman et al.

9 2011, Revell et al. 2011, Thomas and Freckleton 2012), discrete random changes at

10 speciation events (Ingram 2010), or shifts in shared adaptive optima (Uyeda and Harmon

11 2015). However phylogenetic models of trait evolution are ecologically neutral, since they

12 are stochastic models that depend on the independent evolution of each species to be

13 statistically well behaved (Pennell and Harmon 2013). Processes such as competition

14 between species are typically not accounted for. In previous models species interactions have

15 been assumed to generate phenomenological outcomes. For example models may assume rate

16 slowdowns associated with competition among lineages either implicitly by modelling

17 through time (Harmon et al. 2010) or explicitly (Mahler et al. 2010). Several models include

18 clade-wide non-random effects (Felsenstein 1988, Hansen 1997, Price 1997, Harvey \&

19 Rambaut 2000, Freckleton \& Harvey 2006, Bartoszek et al. 2012), reflecting the interaction

20 of species with their environment, but none of these models permits trait values to be

21 influenced by interspecific interactions.

22 Phylogenetic data has been simulated with competitive interactions (Freckleton et al.

23 2003, Nuismer and Harmon 2015). However, direct parameterisation with data is difficult

24 because of the complexity of accounting for interspecific interactions. Niche-filling models

25 of trait evolution on trees (Price 1997, Rambaut and Harvey 2000, Freckleton and Harvey

26 2006) are models of adaptive radiations where new species move discretely to the nearest of a 
1 random set of points (niches) in trait-space. Simulations under these models show that such

2 ecological processes affect inferences drawn from comparative analyses. The most important

3 conclusion from the analysis of such models is that methods based on Brownian motion are

4 inappropriate, or even misleading, when applied to traits evolving in such systems. However

5 the problem of modelling such data has never been satisfactorily resolved (Freckleton and

6 Harvey 2006), largely because of the complexity of statistically describing the traits of a set

7 of interacting species.

8 In terms of fitting complex models to data one potential approach is Approximate

9 Bayesian Computation (ABC; see Beaumont 2010). This provides a simple method for

10 generating posterior probabilities of models, provided we can simulate them. It is therefore

11 well suited to fitting complex models, where it is not possible to compute a likelihood

12 function. In this way species interactions can be incorporated into evolutionary models, thus

13 permitting better inference of the underlying ecology. $\mathrm{ABC}$ has been explored for simple

14 phylogenetic trait evolutionary models (Kutsukake and Innan 2013) including birth-death

15 models (Slater et al. 2012), but its flexibility has not previously been used for including

16 complex effects like interspecific interactions.

17 In this paper we introduce a new model for the evolution of interacting species within

18 phylogenetic data. Our objective is to create a model that includes interactions and makes

19 realistic predictions, but that also may be fitted to real data. The model predictions are

20 compared with those of the Brownian motion and rate-change models for sympatric clades.

21 We then outline how ABC methods may be used to detect competition effects and we show

22 that that the model is readily fitted to data. Finally, we apply these methods to a simple case

23 study, the adaptive radiation of Galapagos finches (Geospiza spp.).

\section{Methods}

\section{The model}


1 Under the Brownian motion (BM) model of trait evolution (Felsenstein 1973), for each

2 species $i$, a trait value $x_{i}$ evolves according to the differential equation,

3

4 where $W(t)$ is the integral of the continuous white noise function, such that over a finite time

5 it has a normal probability distribution: $W(t) \sim N(0, \Delta t)$. The BM model has two free

6 parameters, the evolutionary rate $\sigma$ and the root trait value $x_{\text {root }}(0)$. The expected variance

7 between tips is proportional to the branch length separating them.

Most models for comparative data are based on modifying this model by adding additional parameters (Pagel 1997, Blomberg et al. 2003, Venditti et al. 2010, Eastman et al. 2011, Revell et al. 2012, Thomas and Freckleton 2012). Notably such models assume that the evolutionary trajectories of species traits are independent and do not account for interactions between different species.

Our competition model is based on the BM model, with a term added to account for interspecific interactions. Competition is modelled such that species with similar trait values tend to evolve away from each other, while species with dissimilar trait values have little influence on each other. To achieve this we assume a flat fitness surface for trait values in the absence of other species. In effect we assume that if the trait in question has a one-to-one correspondence with some resource, e.g. body size and prey size, then the distribution of resources is flat. We assume that a species with a given trait value has a corresponding 'ideal' resource but also uses up other resources such that the distribution of resource types used is normal and centred on the 'ideal' resource type. Therefore a Gaussian curve is associated with each species along a single trait axis represents this resource use and consequently its amount of influence on other species as a function of the difference in trait value between the two species (Doebeli and Dieckmann 2003, Pigolotti et al. 2010, Liemar et al. 2013, Liemar et al. 2008).

The repulsion between two species in trait space is assumed to be proportional to the 
1 overlap of each of their associated curves. For the evolution of a single trait $x$ in a species $i$,

2 we get a deterministic term, scaled by a parameter $a$, in addition to the BM term:

$$
d x_{i}=a \sigma \sum_{j} \text { overlap }\left(x_{i}, x_{j}\right) d t+\sigma d W_{i}(t) \quad \text { Equation } 2
$$

4 The area of overlap of two normal curves is related to the cumulative normal function $\Phi$ of

5 minus the distance (in standard deviations) between them, such that the overlap is equal to

$62 \Phi(-$ distance / 2) (Inman and Bradley 1989). The overlap of two curves very far from

7 each other is $2 \Phi(-\infty)=0$, whereas the overlap of two curves with the same centre

8 is $2 \Phi(0)=1$.

9 The relative intensity of competition is measured by the competition parameter $a$ and

10 the Brownian rate parameter $\sigma$ in combination. Ideally we would have chosen to make the

11 kernel width an additional parameter of the model. However in practical terms it would not

12 have been possible to distinguish this effect from that of the competition parameter $a$.

13 Appendix A shows that to a linear approximation the effects of the two are the same, and so

14 they are likely to be statistically indistinguishable.

15 The instantaneous change of the trait value $x_{i}$ of species $i$ is given by equation 3 :

$$
d x_{i}=a \sigma \sum_{j} e_{i j} 2 \Phi\left(-\left|x_{i}-x_{j}\right|\right) d t+\sigma d W_{i}(t)
$$

\section{Equation 3}

17 Each $x_{j}$ is a vector in trait space; the index $j$ denotes species. The right-hand-side has two

18 terms: the first is a deterministic 'competition' term, which pushes apart species that are

19 nearby in trait-space. $e_{i j}$ is the unit vector pointing from species $j$ to species $i$ in trait-space.

20 Thus, $a e_{i j} \Phi\left(-\left|x_{i}-x_{j}\right|\right)$ is a vector in trait-space pointing from species $i$ to species $j$,

21 proportional to the model parameter $a$ and depending on the closeness in trait-space of

22 species $i$ and $j$. What distinguishes this model from previous ones is that in the competition

23 term of the equation all traits are linked: the evolution of two species away from each other in

24 trait space depends on the Euclidian distance between them, as well as their distances to all

25 other species. 
We largely concentrate here on single resources and traits. However, more generally a

2 multivariate normal curve in trait-space may be associated with each species in order to

3 model interactions along several resource axes.

$4 \quad$ In both the BM and competition models, trait variance increases without bound as

5 time progresses. In reality there are limits that will be driven by ecology or by developmental

6 and physiological constraints. We therefore adapted the model by imposing hard limits on

7 trait-space, such that species can evolve up to a chosen extreme value but no further. This

8 model was simulated alongside the limitless model and hence we obtain a new model with

9 constrained trait/niche space. We assume that the limits are symmetric and defined by the

10 most extreme value $L$.

\section{Simulation framework}

13 In a BM model trait evolution may be modelled readily and quickly because species are

14 assumed to be independent. However our model requires that we simulate evolution over

15 interacting branches, which adds to the computational overheads. The approach used here is

16 to take slices of evolutionary time separating speciation events and to apply our model of

17 evolution to each slice sequentially. Within each slice branches are evolved simultaneously.

18 Simulation starts at the root of the tree, with time (branch length) $t$ to the first speciation

19 event. At speciation the trait value of the speciating branch is copied to both of its offspring.

20 The time to the next speciation event is found, and the process is repeated until we have the

21 required tip trait values, including a period between the final speciation event and the tips.

22 Simulations were performed on random ultrametric trees generated under a Yule

23 process (TESS, Hoehna 2013) with 20 to 80 tips. These tree sizes are typical for datasets used

24 to test for evidence of adaptive radiation. Because the competition model is designed for

25 sympatric, interacting sets of species having undergone adaptive radiation, it is unlikely that 
1 numbers of species will be very large. Values of trait variance across tips were calculated as

2 the mean of the tip variances of 1000 trees with the same tip count.

3 The same routine was used to compute the correlation between correlated traits under

4 different competitive regimes, this time with a fixed tree size of 50 tips. We also generated

5 distributions of tip trait values, for Brownian models without competition, and with and

6 without limits. For a single random phylogeny, each evolutionary model was simulated 1000

7 times, and all the tip values pooled to create a frequency distribution. We repeated the same

8 simulations for trees of different sizes (20, 40, 60 and 80 tips) and calculated the variance of

9 each set of tip trait values. This enables the assessment of how the evolutionary model affects

10 the relationship between phylogeny size and trait variance.

\section{Model comparisons and likelihoods}

13 We fitted the model to data using Approximate Bayesian Computation (ABC) (reviewed in

14 Beaumont 2010, Csilléry 2010, Hartig 2011). ABC can be used for comparing the

15 probabilities of datasets under different models when these probabilities are difficult to

16 compute directly. This is because the only requirement to perform $\mathrm{ABC}$ is that we can

17 simulate new datasets using the model. The dataset probabilities are approximated by

18 simulating a large number of datasets, and 'accepting' only those simulations that are very

19 similar to the observed dataset. This similarity can be judged either from the data values

20 themselves, or using summary statistics. The proportion of simulations that are accepted is

21 then assumed to be proportional to the dataset probability. When the model contains

22 continuous parameters, we sample across these parameters and obtain an approximate

23 probability density for the observed data under any point in a range of parameter values. This

24 can be used to estimate the likelihood curves of fitted models. ABC relies on the likelihood

25 being a fairly smooth function of the model parameters (Hartig 2011). 
To apply $\mathrm{ABC}$ to phylogenies (e.g. Beaumont 2010) we sample the parameters of the

2 evolutionary model randomly many times from a prior parameter distribution. Here we

3 choose the prior distribution to be uniform, with the model necessitating a hard limit at zero

4 for both the Brownian rate and the competition strength. For each set of parameters, trait

5 datasets are then simulated for the known phylogeny. Summary statistics are generated for

6 the simulated data, and only those simulations for which the summary statistics are within a

7 small value $\varepsilon$ of the observed data's summary statistics are accepted. Thus, for observed data

$8 \quad D$ and tolerance $\varepsilon$, we accept some simulated data $D^{\prime}$ if

$$
\rho\left(D^{\prime}, D\right)<\varepsilon
$$

\section{Equation 5}

10 where $\rho$ is the discrepancy, or distance in summary statistic space, between $D^{\prime}$ and $D$. In

11 practice we chose the tolerance $\varepsilon$ based on the size of posterior sample that we wanted to

12 obtain, so we might simulate a million datasets and choose $\varepsilon$ such that we accept the best 500

13 simulations. By plotting acceptance rate against parameter values, we get an estimated

14 likelihood surface.

To compare simulated and observed datasets, we need to compare summary statistics.

16 We chose to use three summary statistics: the mean and the variance of the differences

17 between each species and its closest neighbour in trait space, and the overall phylogenetic

18 signal as measured by Blomberg's $K$ (Blomberg et al. 2003). The rationale for using these

19 three was to capture the overall amount of evolution, the overdispersion of trait values, and

20 the phylogenetic structuring of the trait values. There is no well-established procedure for

21 choosing summary statistics for ABC. High sufficiency is needed to compare models, but the

$22 \mathrm{ABC}$ method quickly loses accuracy and stability with large numbers of summary statistics

23 (Csilléry 2010). Our summary statistics were chosen on a pragmatic basis, since they capture

24 the important aspects of the model's behaviour, namely increased divergence between sibling

25 species, and an even overall distribution of traits across the phylogeny. Hartig et al. (2011) 
1 recommend empirical tests of sufficiency by comparing analyses that reject using summary

2 statistics to ones that use the actual data. We performed this test for small trees (5 tips) with

3 simulated datasets, and found that the three summary statistics resulted in power and

4 parameter estimates that were not distinguishable from those produced by using the tip trait

5 values themselves as the summary statistics used to compare models and generate likelihood

6 estimates.

We chose to compare the competition model with the BM model using maximum

8 approximated likelihood, because the BM model is embedded in the competition model. The

9 null and alternative $\mathrm{ABC}$ acceptance rates $A$ give an estimate of the likelihood $L(H \mid D)$ of the

10 observed dataset under the various model parameters, assuming a smooth probability

11 distribution. Then the log-likelihood ratio statistic for the comparison of two models $H_{0}$ and

$12 H_{1}$, when there is no prior difference in model likelihood expectation, is given by:

$$
-2 \log \frac{L\left(H_{1} \mid D\right)}{L\left(H_{0} \mid D\right)}=-2 \log \frac{A_{1}}{A_{0}} .
$$

Equation 6

14 When the models are nested, this test statistic approximates a $\chi$-squared distribution given

15 certain assumptions: large samples and normally distributed parameters. However, these

16 assumptions may be significantly violated by phylogenetic methods (Freckleton 2009). For

17 instance, in the BM model $\sigma$ is bounded at zero, and in our competition model $a$ is also

18 bounded at zero. To correctly interpret the test statistic, therefore, the null distribution of the

19 log-likelihood ratio test statistic was assessed with a parametric bootstrap.

20 The bootstrap was undertaken by performing the model comparison analysis on

21 datasets generated under BM, to create a null distribution of likelihood ratios. Then if, for

22 example, we want to know the likelihood ratio corresponding to a p-value of 0.05 , we simply

23 look at the $95^{\text {th }}$ percentile of the null distribution. The resulting Type I error rate is therefore

24 chosen by design: if a significant likelihood ratio is one that corresponds to a p-value of $5 \%$,

25 then the Type I error rate is 5\%. To estimate typical significance thresholds, we performed 
1 this procedure for random trees using 1000 random datasets.

2 The power to reject BM in favour of the competition model was assessed by using

3 random ultrametric Yule trees (20, 40, 60 and 80 tips). The bootstrap process was performed

4 to determine the significance threshold for that tree. Then, for a given value of the

5 competition parameter $a$, we simulated a large number of datasets and determined the

6 likelihood ratio (between the BM and competition models) for each one. The proportion of

7 these datasets which showed significant support for competition effects defines the power of

8 the model for that value of $a$. We repeated this process for a range of competition strengths

9 from $a=0$ to $a=5$. This range covers evolution from a Brownian process with no

10 interspecific interaction $(a=0)$ to a largely deterministic regime with high phylogenetic

11 structuring of trait values $(a=5)$.

12 To evaluate the simulated data produced by the competition model, other comparative

13 models were fitted to the data: the Brownian model itself, the $\kappa$-model, which measures the

14 degree to which evolution is speciational rather than gradual (Pagel 1997), and K, a measure

15 of phylogenetic signal (Blomberg et al. 2003). Parameter estimates were generated using the

16 R packages geiger (Harmon et al. 2008) and picante (Kembel et al. 2010).

17 The dataset simulations were written in $\mathrm{C}++$. Scripts for using these datasets for

18 likelihood estimation were written in R ( $\mathrm{R}$ Development Core, 2005), using ape (Paradis et

19 al. 2004) and TESS (Hoehna 2013) for tree generation (code available online).

21 Results

\section{Example of clade evolution under the competition model}

23 Illustrative examples of evolution under the competition model are shown in Figure 1.

24 Estimates of phylogenetic signal and of rate-change transformations were generated for these 
1 simulated data and also are shown in Figure 3. The evolution of each species is tracked

2 through time from left to right.

3 Competition tends to increase the overall variance in traits amongst the species in a

4 phylogeny, as is clear from the increase in range and variation of traits moving from Figure

$5 \quad 1 \mathrm{~A}$ to Figure 1D. This is because species experiencing competition from other species are

6 more likely to evolve extreme trait values to become more different and escape competition.

$7 \quad$ As the strength of competition is increased, the differences between species become

8 more clearly defined, with them occupying distinct positions in niche space. There are fewer

9 crossings over of trait's evolutionary paths over time between species, and the phylogenetic

10 signal $K$ exceeds the neutral BM prediction of $K=1$. Competition thus increases

11 phylogenetic signal above that expected under the BM model, while presenting the

12 appearance of a considerable tree-wide evolutionary slowdown. This means that a species'

13 trait values map more directly onto its position in the tree. For sympatric clades, there is thus

14 a prediction of traits being more phylogenetically conserved than under BM.

15 Typical estimates for commonly used branch transformation parameter $\kappa$ from these

16 datasets are also shown in Figure 1. $\kappa$ measures the rate change along branches, and overall

17 measures the degree to which change is speciational (Pagel 1997). A transformation

18 parameter $\delta$ models the overall changes in evolutionary rate across the tree, with lower

19 values corresponding to evolutionary slowdowns (Pagel 1997). We find that the $\delta$ parameter

20 diminishes very rapidly as competition is increased. This reflects an apparent slowdown of

21 evolutionary rate, which becomes more pronounced as the value of $a$ increases. Species

22 competing for unoccupied niche-space thus evolve more rapidly early on in their

23 development, when they are more similar to one another and the effects of competition are

24 stronger, as one would expect in an adaptive radiation (Yoder 2010). 


\section{Trait distributions across tree tips}

2 The distribution of trait values of the phylogeny tips is flattened in the competition model

3 compared with BM models, which predict normal distributions for large trees. This outcome

4 is expected when competition shapes trait values (Davies et al. 2012). Figure 2A shows the

5 model predictions for tip trait value distributions. The impact of competition on trait

6 distributions is even more pronounced where hard limits are placed on the available range of

7 trait values.

8 In addition to creating a more even trait distribution, competition increases the overall

9 amount of trait divergence, given equal BM rates (Figure $2 \mathrm{~B}$ ). This is consistent with the

10 expectation that equivalent species sets should be more diverged in sympatry than in

11 allopatry when there is competition (Schluter 2000). From a biological perspective there is

12 thus a prediction that competition leads to a wider range of morphological variation in a

13 clade, reflecting the increased tendency towards extreme traits when there is lots of

14 competition.

\section{Effects of tree size}

17 We used trees normalised to the same total length, regardless of the number of tips. Under

$18 \mathrm{BM}$ and rate-change models, the variance of tip trait values shows no change with increasing

19 the number of tips (in agreement with Ricklefs 2004). In the competition model larger trees

20 have greater variance, since a greater number of species are 'pushing' each other away; this is

21 shown in Figure 2C. This relationship seems to be approximately linear for the unbounded

22 competition model. When hard limits are imposed, the variance reaches a maximum

23 corresponding to the position of the extremes.

\section{Effects of competition on correlated traits and phylogenetic signal}


1 For pairs of traits, in which the evolutionary changes in trait values are correlated, the

2 correlation between the traits decays rapidly with increasing competition strength. This is

3 even more pronounced when there are limits on extreme trait values. Figure 2D and Figure

$42 \mathrm{E}$ show how the correlation decays. By de-correlating traits, competition forces the trait

5 space to be occupied more evenly. An interesting corollary to this may be an observable

6 pattern of high correlation between traits within species, but reduced correlations between

7 species.

8 Phylogenetic signal is increased by competition as species tend to be remain adjacent

9 in trait space to their close relatives (Figure 2E), since their trait values are unlikely to "cross

10 over' with time. Plots of traits through time therefore become more defined and tree-like.

11 This can be seen for example in the sample simulations of Figure 3. Correlation between

12 traits has little effect on the phylogenetic signal exhibited by the individual traits under either

13 the BM model or the competition model. Limits reduce the phylogenetic signal, since there is

14 less trait-space for distantly related species to diverge. Indeed, without competition driving

15 the signal up (i.e. when $a=0$ ), the model with limits predicts reduced signal compared with

16 the BM model, with $K<1$.

17 Price's (1997) model of adaptive radiations has the feature that when two traits have

18 correlated evolution, the phylogenetically independent contrasts are less strongly correlated

19 than the traits themselves (Price 1997, Rambaut and Harvey 2000, Freckleton and Harvey

20 2006). A Brownian model predicts equal correlation for both traits and contrasts.

21 We compared trait and contrasts correlations under the competition model presented

22 here. Competition tends to reduce correlation between traits, as discussed above, but we set

23 the Brownian evolution of the traits to have very high correlation. The results are shown in

24 Figure 3. In contrast with the result for the Price (1997) model, we found that contrasts had

25 slightly higher correlations than traits. This probably reflects the fact that competition tends

26 to have a greater effect earlier in the evolutionary history of any particular species. 


\section{Power}

3 The power to detect competition effects against a background of BM evolution is shown in

4 Table 1 for trees of various sizes. We define the power as the frequency with which simulated

5 datasets show significant support for competition effects as opposed to the (nested) BM null

6 model. Power is greatest for large trees with high competition strength relative to BM rate.

7 This can be interpreted as the relative contribution to overall evolutionary change of

8 competitive effects versus other, effectively random, effects.

9 In this context a significant dataset is one for which the Type I error rate is estimated

10 to be $\leq 0.05$. This is the frequency with which data from null model simulations display

11 model likelihood ratios that equal or exceed the ratio for the 'observed' dataset. This is

12 determined via a parametric bootstrap.

\section{Case study: Galapagos finches}

15 The simulations described above demonstrate two things: first that the model we describe

16 successfully captures behaviour that we would expect to be observed in systems of

17 interacting species. And second, that it may be applied to data and used to infer the presence

18 of competitive interactions. In order to use the model in a real-world example we applied the

19 competition model to an example dataset, using trait measurements collected in Harmon et al.

20 (2010a; originally Grant and Grant 2002, Lack 1947; repository in Harmon 2010b

21 http://dx.doi.org/10.5061/dryad.f660p), and a recent molecular phylogeny (Lamichhaney et

22 al. 2015). We used the Galapagos finches (Geospiza spp.), since they are a well-studied

23 adaptive radiation, and ecological effects were anticipated to be of importance. The effect of

24 character displacement on intraspecific variation among these finches is well documented

25 (e.g. Grant and Grant 2006). Here we are looking to see whether the same mechanism has 
1 had an effect on the overall distribution of traits across the clade.

\section{Methods}

4 The phylogeny of Galapagos finches was taken from Lamichhaney et al. (2015). Data was

5 available for five traits: wing length, tarsus length, bill length (culmen), bill depth and bill

6 width (gonys) (Harmon 2010b). We computed likelihood ratios for each trait individually.

$7 \quad$ After simulating data on the phylogeny to determine likelihood cutoffs for rejecting

8 BM, a likelihood comparison between the competition model and the nested BM model was

9 run for each of the five traits separately. We performed the tests twice, once including and

10 once excluding the phylogenetic summary statistic $K$, to judge the importance of signal in

11 favouring the competition model. Applying the bootstrap process for single traits gave a

12 likelihood ratio for the full tree of 3.49 corresponding to $\mathrm{p}=0.05$, while 3.79 corresponded to

$13 \mathrm{p}=0.01$. There is one degree of freedom difference between the competition and BM models.

\section{Results}

16 The parameter estimates and model likelihood ratios are shown in Table 2. The beak shape

17 traits showed greater support for competition compared with Brownian evolution than the

18 other traits. This appears to point to an ecological effect: the competition model implies a

19 tendency towards well-differentiated niches that don't cross, and the beak shape is an

20 'ecological' trait, in the sense that it corresponds strongly to feeding habits (Grant and Grant

21 2011). Figure 4 shows illustrative plots of simulated trait evolution using the model

22 parameters that were estimated for the culmen length. Compared with BM, shown in Figure

$234 \mathrm{~A}$, the tree becomes very well defined, with strong phylogenetic signal.

24 One of the beak traits, culmen length, favoured the competition model when signal

25 was not used, but less so when it was included. Brownian rate parameter estimates under the 
1 model with limits are higher than those for the non-limited competition model. This higher

2 rate does not result in a greater total amount of evolutionary change, due to the hard limits

3 that are reached either way. This result does, however, suggest that the niche landscape may

4 be the limiting factor in the finches' evolution: the finch traits are capable of evolving

5 rapidly, but their values are constrained by the combination of interactions between species

6 and environmental limits on niche space.

7 It is worth noting that none of these results takes into account intraspecific variation

8 or uncertainty in the finch phylogeny. Species interactions will be somewhat independent on

9 different islands, as illustrated by the character displacement seen in intraspecific trait

10 variation (Grant and Grant 2006). Furthermore, the tree topology is fairly uncertain, since

11 many of the tree node dates have error bounds that are large compared with the distances

12 between nodes (Lamichhaney et al. 2015).

\section{Discussion}

15 There have been several recent approaches to integrating ecological processes into

16 phylogenetic models of evolution (Cavender-Bares et al. 2009, Hadfield et al. 2013, Pennell

17 and Harmon 2013). As a step in this direction, we have created a model of interspecific

18 competition on phylogenies of coexisting species. The model provides a process-based

19 picture of competitive evolution, linking statistical patterns directly to the underlying

20 ecology. It generates the patterns we expect to see in situations where interspecific

21 competition is important.

22 Competition and niche overlap have a complex relationship, and the former may

23 cause or inhibit the latter depending on the particular niche being measured. According to one

24 possibility, there is an optimum position on a niche axis, and species will compete with each 
1 other to occupy it. Consequently they will evolve similar trait values tailored to that

2 optimum (Colwell and Futuyma 1971). Another possibility is that the niche is represented by

3 a continuum of equal fitness along the axis. In this case species will compete for empty

4 regions of the niche axis and evolve minimally-overlapping, evenly-spaced trait values,

5 consistent with the ecological idea of character displacement (Grant 1972, Strong et al. 1978,

6 Dayan and Simberloff 2005). Our competition model accommodates the latter in a

7 phylogenetic context.

8 One prediction of the competition model is a flattened distribution of trait values

9 among contemporary species of a single sympatric clade, in common with several previous

10 models. Indeed, competition is often inferred from such evenness (Dayan and Simberloff

11 2005, Davies et al. 2012). The same pattern can however be caused by competition at the

12 community assembly level rather than in situ trait evolution (Cavender-Bares et al. 2009,

13 Stuart and Losos 2013), or by geographical structure in speciation and extinction (Pigot and

14 Etienne 2015). For this reason the model presented here is applicable only to clades known to

15 share a common environment, or subsets of clades formed by restricting attention to a single

16 environment. Since the model incorporates pairwise interactions between all the species

17 present, it requires that each species be at least somewhat sympatric with each other species.

18 Strong niche-conservatism is then predicted for sympatric clades. However, it is worth

19 commenting that species within a clade are not exclusively sympatric or allopatric in their

20 distributions. Across clades there is a wealth of variation in species' geographic structure and

21 opportunity to interact (Fitzpatrick et al. 2008). Taking variation in range overlaps into

22 account may be an interesting future development in such modelling.

23 The pattern of non-Brownian value distributions and high phylogenetic signal is also

24 generated by an alternative, but related, mechanism, where instead of a continuously

25 available space of niches, niches are discrete, with one species per niche, and new species

26 arise in nearby discrete niches (Price 1997, 2014). Determining a method to distinguish this 
model will be a key advance. Specifically it differs from the current model in that the tree topology is a function of trait values and not fixed as in our model.

Most phylogenetic models of trait evolution are modifications of the random BM model. As noted above, adaptive radiations are generally consistent with a tree-wide gradual slowdown in evolutionary rate (delta-model: Pagel 1997; ACDC model: Blomberg et al. 2003). Speciational evolution can be modelled as a gradual branch-wise slowdown ( $\kappa$ model, Pagel 1997), or by partitioning evolution into gradual and speciational parts (Bokma 2008, Ingram 2011). Discrete shifts in evolutionary rate can be modelled to detect, for example, adaptive radiations embedded in a larger tree (O’Meara et al. 2006, Thomas et al. 2006). Slowdowns in evolutionary rate have also been observed as a function not of time but directly of a clade's size (Mahler et al. 2010). The results for our competition model suggest that it reproduces the appearance of a strong tree-wide slowdown. During a radiation, though, competition is predicted to cause overall trait variance to increase much more rapidly. Our results for the competition model also demonstrate raised phylogenetic signal, in agreement with similar results in Nuismer and Harmon (2015). In all analyses we used a fixed competition kernel width. The fact that this width is not distinguishable from the competition strength itself suggests that the amount of variation possible within a single niche is not readily ascertained from a phylogeny and trait data. Measurements of intraspecific variation will be more suited to this question. In fact, the competition kernel widths could be set empirically before analysis, if data on intraspecific variation were available.

Our results for the Galapagos finches support the well-known presence of character displacement in that clade (Grant and Grant 2006), and further suggest that interspecific competition is a significant force comparable with other, effectively random, sources of evolutionary change for the Galapagos finches. For some beak shape traits, the Galapagos finches exhibit the elevated phylogenetic signal predicted by the competition model. 
The usefulness of the model in detecting competition in other datasets will depend on

2 the strength of competition effects in nature. The model could readily accommodate known

3 extinct species, but unknown extinct species may affect the contemporary datasets generated

4 by the model, since we will not be accounting for their past interactions with extant species.

5 Additionally, because it involves between-species interactions, the model is particularly

6 sensitive to missing data, both species themselves and their trait values.

7 There are numerous speciation/extinction models for phylogenies (Nee et al. 1994,

8 Pybus \& Harvey 2000, Rabosky 2006, Freckleton et al. 2008, FitzJohn 2010), including some

9 that are expected to correspond to clades with interspecific competition (Harmon et al 2010,

10 Etienne et al. 2012). Our model is concerned only with trait evolution. Trait evolution and

11 diversification rates may be coupled in nature, however, and may both vary with factors such

12 as interspecific competition. Building models of adaptive radiations that simultaneously

13 predict trait evolution and diversification will be key in the future.

14 As phylogenetic methods continue to be used to infer evolutionary processes, it will

15 be important to include specific ecological mechanisms (Vamosi et al. 2009). Competition

16 for ecologically distinct roles is often implicitly or explicitly assumed in adaptive radiations,

17 but its prevalence and importance remain uncertain (Schluter 2000, Stuart and Losos 2013).

18 We have developed an explicit model of competition on phylogenies, to detect competitive

19 effects in sympatric adaptive radiations, and to enable measurement of competition strength.

20 The predictions of this model may help to understand the roles ecological processes play in

21 shaping trait evolution.

\section{Literature Cited}

24 Abrams, P. 1983. The theory of limiting similarity. Annual Review of Ecology and Systematics 14:359-376. 
1 Beaumont, M. A. 2010. Approximate Bayesian computation in evolution and ecology.

2 Annual Review of Ecology, Evolution, and Systematics 41:379-406.

3 Blomberg, S. P., T. Garland, and A. R. Ives. 2003. Testing for phylogenetic signal in

4 comparative data: behavioral traits are more labile. Evolution 57:717-745.

5 Bokma, F. 2008. Detection of "punctuated equilibrium" by Bayesian estimation of speciation

6 and extinction rates, ancestral character states, and rates of anagenetic and cladogenetic

7 evolution on a molecular phylogeny. Evolution 62:2718-2726.

8 Cavender-Bares, J., K. H. Kozak, P. V. A. Fine, and S. W. Kembel. 2009. The merging of

$9 \quad$ community ecology and phylogenetic biology. Ecology Letters 12:693-715.

10 Colwell, R. K., and D. J. Futuyma. 1971. On the measurement of niche breadth and overlap.

$11 \quad$ Ecology 567-576.

12 Davies, T. J., N. Cooper, J. A. F. Diniz-Filho, G. H. Thomas, and S. Meiri. 2012. Using

13 phylogenetic trees to test for character displacement: a model and an example from a

14 desert mammal community. Ecology 93:S44-S51.

15 Dayan, T., and D. Simberloff. 2005. Ecological and community-wide character displacement:

16 the next generation. Ecology Letters 8:875-894.

17 De Mazancourt, C., E. Johnson, and T. G. Barraclough. 2008. Biodiversity inhibits species'

18 evolutionary responses to changing environments. Ecology Letters 11:380-388.

19 Doebeli, M., and U. Dieckmann. 2003. Speciation along environmental gradients. Nature

$20 \quad 421: 259-264$.

21 Eastman, J. M., M. E. Alfaro, P. Joyce, A. L. Hipp, and L. J. Harmon. 2011. A novel

22 comparative method for identifying shifts in the rate of character evolution on trees.

$23 \quad$ Evolution 65:3578-3589.

24 Emerson, B. C., and R. G. Gillespie. 2008. Phylogenetic analysis of community assembly and

25 structure over space and time. Trends in Ecology \& Evolution 23:619-630.

26 Etienne, R. S., and J. Rosindell. 2012. prolonging the past counteracts the pull of the present: 
protracted speciation can explain observed slowdowns in diversification. Systematic

$2 \quad$ Biology 61:204-213.

3 Felsenstein, J. 1973. Maximum-likelihood estimation of evolutionary trees from continuous

4 characters. American Journal of Human Genetics 25:471-492.

5 Felsenstein, J. 1985. Phylogenies and the comparative method. The American Naturalist

$6 \quad 125: 1-15$

7 FitzJohn, R. G. 2010. Quantitative traits and diversification. Systematic Biology 59:619-633.

8 Fitzpatrick, B. M., J. A. Fordyce, and S. Gavrilets. 2008. What, if anything, is sympatric

$9 \quad$ speciation? Journal of Evolutionary Biology 21:1452-1459.

10 Freckleton, R. P. 2009. The seven deadly sins of comparative analysis. Journal of

$11 \quad$ Evolutionary Biology 22:1367-1375.

12 Freckleton, R. P., and P. H. Harvey. 2006. Detecting non-brownian trait evolution in adaptive

13 radiations. PLoS Biology 4:e373.

14 Freckleton, R. P., P. H. Harvey, and M. Pagel. 2002. Phylogenetic analysis and comparative

15 data: a test and review of evidence. The American Naturalist 160:712-726.

16 Freckleton, R. P., M. Pagel, and P.H. Harvey. 2003. Comparative methods for adaptive

17 radiations. Pages 391-407 in T. M. Blackburn and K. J. Gaston, eds. Macroecology:

18 concepts and consequences. Blackwell Scientific, Oxford.

19 Freckleton, R. P., A. B. Phillimore, and M. Pagel. 2008. Relating traits to diversification: a

20 simple test. The American Naturalist 172:102-115.

21 Garland, T., P. H. Harvey, and A. R. Ives. 1992. Procedures for the analysis of comparative

22 data using phylogenetically independent contrasts. Systematic Biology 41:18-32.

23 Gillespie, R. G., F. G. Howarth, and G. K. Roderick. 2001. Adaptive radiation. Encyclopedia

24 of Biodiversity 1:25-44.

25 Grant, P. R., and B. R. Grant. 2002. Unpredictable evolution in a 30-year study of Darwin's

26 finches. Science 296:707-711. 
1 Grant, P. R., and B. R. Grant. 2006. Evolution of character displacement in Darwin's finches.

$2 \quad$ Science 313:224-226.

3 Grant, P. R., and B. R. Grant. 2011. How and why species multiply: the radiation of darwin's $4 \quad$ finches. Princeton University Press, Princeton.

5 Hadfield, J. D., B. R. Krasnov, R. Poulin, and S. Nakagawa. 2014. A tale of two phylogenies:

6 comparative analyses of ecological interactions. The American Naturalist 183:174-187.

7 Hansen, T. F. 1997. Stabilizing selection and the comparative analysis of adaptation.

$8 \quad$ Evolution 51:1341-1351.

9 Harmon, L. J., J. B. Losos, T. Jonathan Davies, R. G. Gillespie, J. L. Gittleman, W. B.

Harmon, L. J., J. B. Losos, T. Jonathan Davies, R. G. Gillespie, J. L. Gittleman, W. B. Jennings, K. H. Kozak, M. A. McPeek, F. Moreno-Roark, T. J. Near, A. Purvis, R. E. Ricklefs, D. Schluter, J. A. Schulte, O. Seehausen, B.L. Sidlauskas, O. Torres-Carvajal, J. T. Weir, A. O. Mooers. 2010a. Early bursts of body size and shape evolution are rare in comparative data. Evolution 64:2385-2396.

Hartig, F., J. M. Calabrese, B. Reineking, T. Wiegand, and A. Huth. 2011. Statistical $14: 816-827$.

Harvey, P. H., and A. Rambaut. 2000. Comparative analyses for adaptive radiations. 
1605.

2 Hoehna, S. 2013. Fast simulation of reconstructed phylogenies under global, time-dependent birth-death processes. Bioinformatics 29:1367-1374

4 Huelsenbeck, J. P., and K. A. Crandall. 1997. Phylogeny estimation and hypothesis testing using maximum likelihood. Annual Review of Ecology and Systematics 28:437-466.

6 Ingram, T. 2011. Speciation along a depth gradient in a marine adaptive radiation. Proceedings of the Royal Society B: Biological Sciences 278.1705:613-618

8 Inman, H. F., and E. L. Bradley. 1989. The overlapping coefficient as a measure of agreement between probability distributions and point estimation of the overlap of two normal densities. Communications in Statistics - Theory and Methods 18:3851-3874.

Johnson, M. T. J., and J. R. Stinchcombe. 2007. An emerging synthesis between community ecology and evolutionary biology. Trends in Ecology \& Evolution 22:250-257.

Kembel, S. W., P. D. Cowan, M. R. Helmus, W. K. Cornwell, H. Morlon, D. D. Ackerly, S. P. Blomberg, et al. 2010. Picante: R tools for integrating phylogenies and ecology. Bioinformatics 26:1463-1464.

Kraft, N. J. B., W. K. Cornwell, C. O. Webb, and D. D. Ackerly. 2007. Trait Evolution, Community Assembly, and the Phylogenetic Structure of Ecological Communities. The American Naturalist 170:271-283.

Kutsukake, N., and H. Innan. 2013. Simulation-based likelihood approach for evolutionary models of phenotypic traits on phylogeny. Evolution 67:355-367.

Lack, D. 1947. The significance of clutch size. Ibis 89.2:302-352.

22 Leimar, O., M. Doebeli, and U. Dieckmann. 2008. Evolution of phenotypic clusters through 23 competition and local adaptation along an environmental gradient. Evolution 62:807822. 
13.

2 Mahler, D. L., L. J. Revell, R. E. Glor, and J. B. Losos. 2010. Ecological opportunity and the rate of morphological evolution in the diversification of greater antillean anoles. Evolution 64:2731-2745.

5 Mayfield, M. M., and J. M. Levine. 2010. Opposing effects of competitive exclusion on the

$6 \quad$ phylogenetic structure of communities. Ecology Letters 13:1085-1093.

7 Moen, D. S., and J. J. Wiens. 2009. Phylogenetic evidence for competitively driven

8 divergence: body-size evolution in caribbean treefrogs (hylidae: osteopilus). Evolution

$9 \quad 63: 195-214$.

10 Monroe, M. J. 2012. Does competition drive character differences between species on a

11 macroevolutionary scale? Journal of Evolutionary Biology 25:2341-2347.

12 Nee, S., E. C. Holmes, R. M. May, and P. H. Harvey. 1994. Extinction rates can be estimated

13 from molecular phylogenies. Philosophical Transactions of the Royal Society B:

14 Biological Sciences 344:77-82.

15 Nuismer, S. L., and L. J. Harmon. 2015. Predicting rates of interspecific interaction from 16 phylogenetic trees. Ecology Letters 18:17-27.

17 O’Meara, B. C., C. Ané, M. J. Sanderson, and P. C. Wainwright. 2006. Testing for different

18 rates of continuous trait evolution using likelihood. International Journal of Organic

19 Evolution 60:922-933.

20 Pagel, M. 1997. Inferring evolutionary processes from phylogenies. Zoologica Scripta

$21 \quad 26: 331-348$.

22 Paradis, E., J. Claude, and K. Strimmer. 2004. APE: analyses of phylogenetics and evolution 23 in R language. Bioinformatics 20:289-290.

24 Parnell, N. F., and J. T. Streelman. 2011. The macroecology of rapid evolutionary radiation.

25 Proceedings of the Royal Society of London B: Biological Sciences rspb20101950.

26 Pennell, M. W., and L. J. Harmon. 2013. An integrative view of phylogenetic comparative 
methods: connections to population genetics, community ecology, and paleobiology. Annals of the New York Academy of Sciences 1289:90-105.

3 Petren, K., B. R. Grant, and P. R. Grant. 1999. A phylogeny of Darwin's finches based on

4 microsatellite DNA length variation. Proceedings of the Royal Society of London B:

$5 \quad$ Biological Sciences 266:321-329.

6 Pfennig, D. W., and K. S. Pfennig. 2010. Character displacement and the origins of diversity.

$7 \quad$ The American Naturalist 176:S26-S44.

8 Price, T. 1997. Correlated evolution and independent contrasts. Philosophical Transactions of

$9 \quad$ the Royal Society B: Biological Sciences 352:519-529.

10 Pigolotti, S., C. López, E. Hernández-García, and K. H. Andersen. 2010. How Gaussian

11 competition leads to lumpy or uniform species distributions. Theoretical Ecology 3:89-

1296.

13 Purvis, A. 2004. Evolution: How do characters evolve? Nature 432.7014.

14 Rabosky, D. L. 2006. Likelihood methods for detecting temporal shifts in diversification $15 \quad$ rates. Evolution 60:1152-1164.

16 Revell, L. J., L. J. Harmon, and D. C. Collar. 2008. Phylogenetic signal, evolutionary 17 process, and rate. Systematic Biology 57:591-601.

18 Revell, L. J., D. L. Mahler, P. R. Peres-Neto, and B. D. Redelings. 2012. A new phylogenetic 19 method for identifying exceptional phenotypic diversification. Evolution 66:135-146.

20 Rice, A. M., and D. W. Pfennig. 2007. Character displacement: in situ evolution of novel

21 phenotypes or sorting of pre-existing variation? Journal of Evolutionary Biology $22 \quad 20: 448-459$.

23 Schluter, D. 2000. Ecological character displacement in adaptive radiation. The American $24 \quad$ Naturalist 156:S4-S16.

25 Schluter, D., and J. D. McPhail. 1992. Ecological character displacement and speciation in 26 sticklebacks. The American Naturalist 140:85-108. 
1 Schoener, T. W. 2011. The Newest Synthesis: Understanding the interplay of evolutionary

2 and ecological dynamics. Science 331:426-429.

3 Sexton, J. P., P. J. McIntyre, A. L. Angert, and K. J. Rice. 2009. Evolution and ecology of

4 species range limits. Annual Review of Ecology, Evolution, and Systematics 40:415-

5436.

6 Slater, G. J., L. J. Harmon, D. Wegmann, P. Joyce, L. J. Revell, and M. E. Alfaro. 2012.

$7 \quad$ Fitting models of continuous trait evolution to incompletely sampled comparative data

8 using approximate bayesian computation. Evolution 66:752-762.

9 Stuart, Y. E., and J. B. Losos. 2013. Ecological character displacement: glass half full or half

10 empty? Trends in Ecology \& Evolution 28:402-408.

11 Team, R Core. 2015. R: A language and environment for statistical computing. Vienna,

$12 \quad$ Austria. https://ww.R-project.org/

13 Thomas, G. H., and R. P. Freckleton. 2012. MOTMOT: models of trait macroevolution on

14 trees. Methods in Ecology and Evolution 3:145-151.

15 Thomas, G. H., R. P. Freckleton, and T. Székely. 2006. Comparative analyses of the

16 influence of developmental mode on phenotypic diversification rates in shorebirds.

17 Proceedings of the Royal Society B: Biological Sciences 273:1619-1624.

18 Tyler, C. L., and L. R. Leighton. 2011. Detecting competition in the fossil record: support for

19 character displacement among Ordovician brachiopods. Palaeogeography,

20 Palaeoclimatology, Palaeoecology 307:205-217.

21 Vamosi, S. M., S. B. Heard, J. C. Vamosi, and C. O. Webb. 2009. Emerging patterns in the

22 comparative analysis of phylogenetic community structure. Molecular Ecology 18:572-

$23 \quad 592$.

24 Webb, C. O., D. D. Ackerly, M. A. McPeek, and M. J. Donoghue. 2002. Phylogenies and

25 community ecology. Annual Review of Ecology and Systematics 33:475-505.

26 Yoder, J. B., E. Clancey, S. Des Roches, J. M. Eastman, L. Gentry, W. Godsoe, T. J. Hagey, 

not certified by peer review) is the author/funder. All rights reserved. No reuse allowed without permission. Evolutionary Biology 23:1581-1596. 


\section{Appendix: Estimating competition strength and effect width}

\section{2 simultaneously}

3 The overlap between species $i$ and $j$ is proportional to $\Phi\left(\frac{-\Delta X_{i j}}{\sigma}\right)$ where $\Phi$ is the cumulative

4 normal distribution. Integrating by parts yields the following approximate function for the

5 overlap between two species:

$6 \Phi\left(\frac{-\left|\Delta X_{i j}\right|}{\sigma}\right)=0.5-\frac{1}{\sqrt{2 \pi}} e^{\frac{-\left|\Delta X_{i j}\right|^{2}}{2 \sigma^{2}}}\left[\frac{\left|\Delta X_{i j}\right|}{\sigma}+\frac{\left|\Delta X_{i j}\right|^{3}}{3 \sigma^{3}}+\cdots\right]$

Equation A1

7 To get the evolutionary rate we multiply this by $a$, giving:

$8 \quad \mathrm{a} \Phi\left(\frac{-\left|\Delta X_{i j}\right|}{\sigma}\right)=0.5 a-\frac{a}{\sqrt{2 \pi}} e^{\frac{-\left|\Delta X_{i j}\right|^{2}}{2 \sigma^{2}}}\left[\frac{\left|\Delta X_{i j}\right|}{\sigma}+\frac{\left|\Delta X_{i j}\right|^{3}}{3 \sigma^{3}}+\cdots\right]$

Equation A2

9 At first glance it might appear that changing $a$ and $\sigma$ would have different effects because the

10 former changes evolutionary rates in a linear manner, whilst the effect of the latter is non-

11 linear. However, if there are a large number of species within a limited niche space, then

12 distances between species will be low, i.e. $\left|\Delta X_{i j}\right|$ is small. Consequently, we can use the

13 following approximation by the Maclaurin series expansion of $e^{x}$ :

$$
e^{\frac{-\left|\Delta X_{i j}\right|^{2}}{2 \sigma^{2}} \approx 1-\frac{1}{2 \sigma^{2}}\left|\Delta X_{i j}\right|^{2}}
$$

14 Substituting into equation (2) and ignoring higher than squared terms we get:

$15 \mathrm{a} \Phi\left(\frac{-\left|\Delta X_{i j}\right|}{\sigma}\right) \approx 0.5 a-\frac{a}{\sqrt{2 \pi}}\left(1-\frac{1}{2 \sigma^{2}}\left|\Delta X_{i j}\right|^{2}\right)\left[\frac{\left|\Delta X_{i j}\right|}{\sigma}\right] \approx 0.5 a\left(1-\frac{\left|\Delta X_{i j}\right|}{\sigma^{2} \sqrt{2 \pi}}\right) \quad$ Equation $A 1$

16 Overall, the rate of evolution is given by the overlap, $2 \Phi\left(\frac{-\Delta X_{i j}}{\sigma}\right)$ multiplied by $a$, yielding:

$$
2 \mathrm{a} \Phi\left(\frac{-\left|\Delta X_{i j}\right|}{\sigma}\right) \approx a\left(1-\frac{\left|\Delta X_{i j}\right|}{\sigma^{2} \sqrt{2 \pi}}\right)
$$

17 This equation is linear in both $a$ and $\sigma^{-2}$. Thus from a statistical perspective $a$ and $\sigma$ will be

18 non-identifiable if the species are interacting strongly. If species are not interacting strongly,

19 i.e. $\Delta X_{i j}$ is large, then the data will contain no information on interactions between species 
bioRxiv preprint doi: https://doi.org/10.1101/033647; this version posted December 4, 2015. The copyright holder for this preprint (which was not certified by peer review) is the author/funder. All rights reserved. No reuse allowed without permission.

1 and hence it will not be possible to fit the model and we cannot estimate either $a$ or $\sigma$.

2

3 
1 Table 1. Power to detect competition versus Brownian Motion as tree size, the Brownian

2 parameter $(\sigma)$ and intensity of competition $(a)$ are varied.

\begin{tabular}{|c|c|c|c|c|c|}
\hline Tree size & $\boldsymbol{\sigma}$ & $a=2$ & $a=4$ & $a=6$ & $a=8$ \\
\hline 20 & 2 & 0.04 & 0.10 & 0.12 & 0.15 \\
\hline 40 & 2 & 0.24 & 0.25 & 0.46 & 0.36 \\
\hline 60 & 2 & 0.44 & 0.46 & 0.58 & 0.45 \\
\hline 80 & 2 & 0.35 & 0.50 & 0.57 & 0.67 \\
\hline 20 & 1.5 & 0.10 & 0.18 & 0.22 & 0.25 \\
\hline 40 & 1.5 & 0.24 & 0.29 & 0.36 & 0.39 \\
\hline 60 & 1.5 & 0.40 & 0.40 & 0.60 & 0.66 \\
\hline 80 & 1.5 & 0.39 & 0.42 & 0.69 & 0.73 \\
\hline 20 & 1 & 0.19 & 0.27 & 0.38 & 0.40 \\
\hline 40 & 1 & 0.26 & 0.54 & 0.47 & 0.54 \\
\hline 60 & 1 & 0.27 & 0.42 & 0.62 & 0.70 \\
\hline 80 & 1 & 0.31 & 0.76 & 0.83 & 0.79 \\
\hline 20 & 0.5 & 0.10 & 0.26 & 0.30 & 0.43 \\
\hline 40 & 0.5 & 0.37 & 0.48 & 0.66 & 0.85 \\
\hline 60 & 0.5 & 0.50 & 0.66 & 0.79 & 0.84 \\
\hline 80 & 0.5 & 0.48 & 0.80 & 0.90 & 0.92 \\
\hline
\end{tabular}

$3 \overline{\text { Note: Because the test is based on a parametric bootstrap, necessarily power }=0.05}$ when $\mathrm{a}=0$,

4 i.e. Brownian motion. 
1 Table 2: Traits and likelihood ratios for model comparisons for Galapagos finches.

(a) Model without limits $\quad$ Model fitted without K $\quad$ Model fitted with K

\begin{tabular}{lllllll} 
Trait & $\boldsymbol{\sigma}$ & $\mathbf{a}$ & $\mathbf{L R}$ & $\boldsymbol{\sigma}$ & $\mathbf{a}$ & LR \\
\hline Wing length & 1.40 & 0.68 & 1.20 & 1.32 & 0.68 & 1.15 \\
Tarsus length & 1.80 & 0.64 & 1.11 & 1.80 & 0.64 & 1.05 \\
Culmen length & 0.60 & 5.08 & $5.12^{*}$ & 0.92 & 2.76 & 3.40 \\
Beak depth & 1.76 & 5.08 & 1.58 & 1.36 & 4.92 & 2.07 \\
Gonys width & 1.76 & 1.00 & 1.75 & 1.64 & 3.40 & 1.83 \\
\hline
\end{tabular}

(b) Model with limits

Model fitted without K Model fitted with K

\begin{tabular}{lllllll} 
Trait & $\boldsymbol{\sigma}$ & $\mathbf{a}$ & $\mathbf{L R}$ & $\boldsymbol{\sigma}$ & $\mathbf{a}$ & $\mathbf{L R}$ \\
\hline Wing length & 2.24 & 0.84 & 1.09 & 1.88 & 1.56 & 1.49 \\
Tarsus length & 2.28 & 0.80 & 0.97 & 2.76 & 1.52 & 1.29 \\
Culmen length & 3.24 & 5.04 & $4.01 *$ & 2.24 & 5.36 & $3.81^{*}$ \\
Beak depth & 1.92 & 5.20 & 2.78 & 1.68 & 5.16 & 3.22 \\
Gonys width & 1.76 & 5.28 & 2.23 & 1.88 & 5.16 & 2.28 \\
\hline
\end{tabular}

2 Note: the finch trait dataset is that given in Lamichhaney et al. (2015). The competition

3 model is compared with the nested BM model. The competition model has one extra

4 parameter compared with the BM model.

5

6 

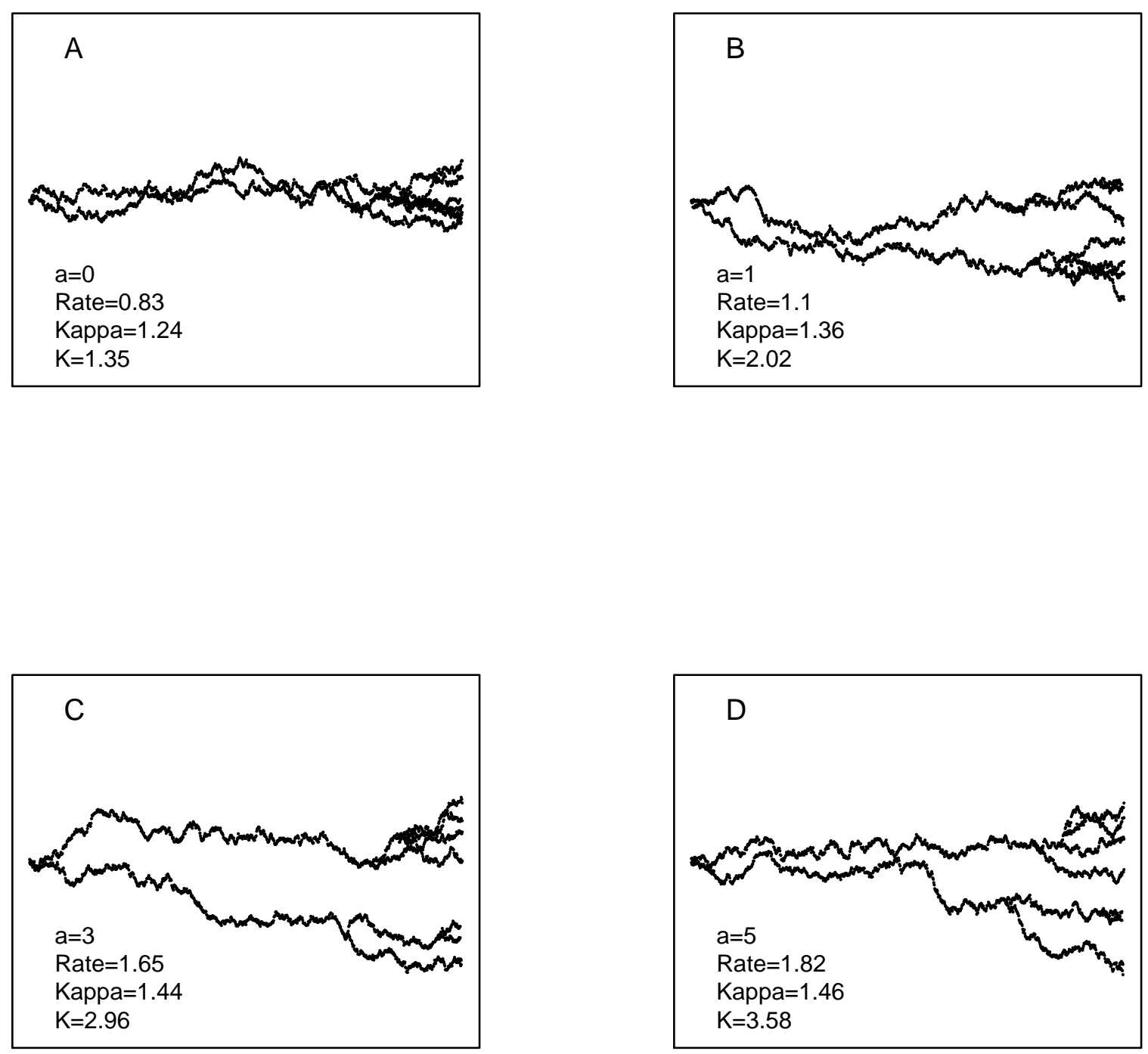

Figure 1. Examples of a single trait evolving under the competition model with different strengths of the competition effect parameter $a$. The parameter values listed by each plot are the averaged estimates under those respective models for several datasets generated under the competition model. When $a=0$ we recover the nested BM model. 

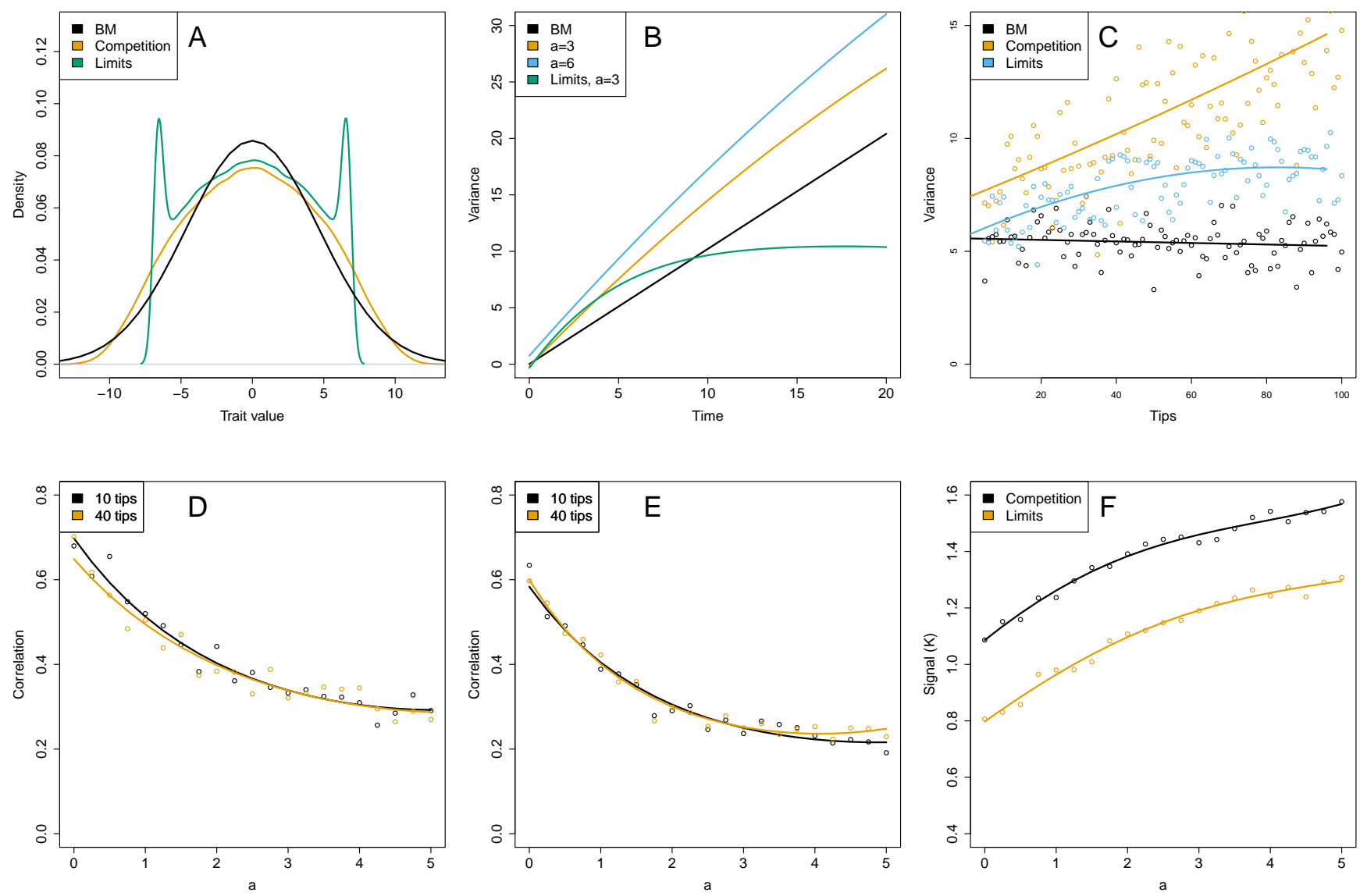

Figure 2. A, B, Effects of interspecific competition on trait value distributions across a clade. The BM model results in a normal distribution, whose variance increases linearly with time. The competition model predicts a flattened distribution, whose variance increases with time, initially faster than for BM, but slowly slowing to the BM rate. When hard limits are placed on trait space, the competitive distribution is further flattened, with probability peaks at the limits, since competition will tend to push species to those limits. C, Effect of tree size on variance of trait values of an evolving clade. Under BM the tree size has no effect, but with competition across the clade, a more numerous clade results in a greater amount of trait variation in that clade. $\mathrm{D}$, The correlation between two traits across the species in a clade, as a function of the strength of interspecific competition parameter. The BM rate parameter in set to 1 throughout. E, Correlation for the model with limits. F, The signal (Blomberg's $K$ ) as a function of competition strength. The traits are evolving with their random (BM) evolution strongly correlated; the pressure from competition to be dissimilar acts against this natural correlation. 

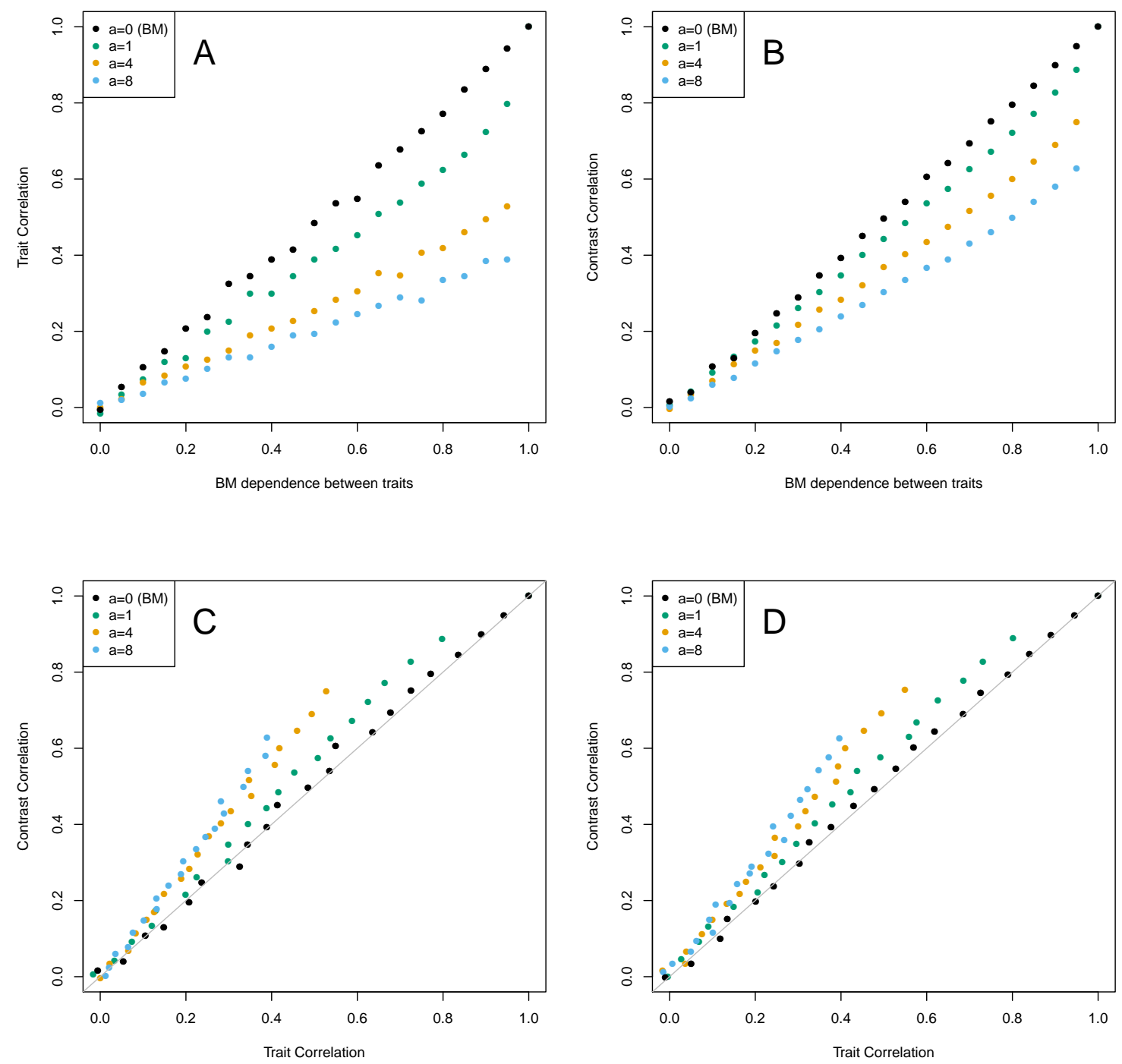

Figure 3. Traits and contrasts for two traits, where one trait has a dependence on the other. For each step in time, the dependent trait has an evolutionary change that depends on the change to the other trait. If the dependence is 1 , then these changes are equal; if the dependence is 0.5 , then the change in the dependent trait is 0.5 of the change in the other trait, and the remaining change is random. A, Trait correlation as a function of the intrinsic trait dependence. B, Contrast correlation as a function of the intrinsic trait dependence. C, Correlation between contrasts is slightly greater than correlation between traits for competitive evolution. D, Contrast and trait correlations for a model of competition with traitspace limits. 


\section{A}
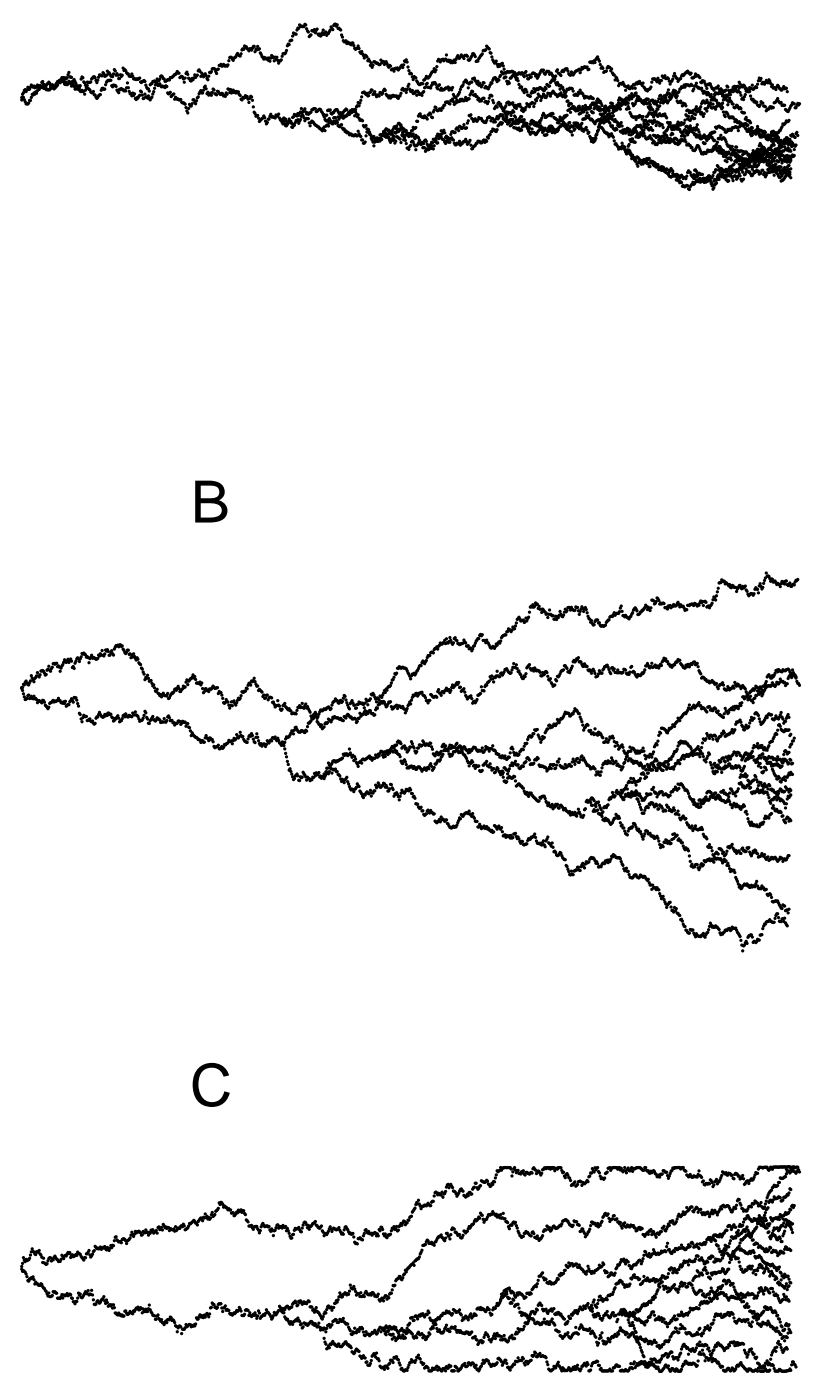

Figure 4. Example simulations of univariate trait evolution on the Darwins finch phylogeny, using model parameters estimated from culmen length data. A, Evolution under Brownian motion. B, Evolution including the competition parameter. C, Evolution including competition and hard limits. 